\title{
UVA/Riboflavin Cross-Linking as an Alternative Treatment for Therapeutic Keratoplasty in Corneal Melting
}

\author{
N Al-Sabai, C Koppen, MJ Tassignon
}

\begin{abstract}
Purpose: To demonstrate the effectiveness of UVA/riboflavin cross-linking $(\mathrm{CXL})$ in the treatment of therapy resistant corneal melting of different etiology.

Methods: This paper describes a series of four cases presenting severe ulcerative keratitis with infectious and noninfectious causes. In spite of intensive medical treatment, corneal melting did develop in all four eyes. CXL was performed to avoid imminent corneal perforation.
\end{abstract}

Results: After CXL, there was a rapid improvement in two eyes which healed completely within a month. In the other two eyes, an emergency keratoplasty had to be performed.

Conclusion: This case series highlights the effectiveness of CXL in the treatment of corneal melting as an addition to our current therapeutic armamentarium. Improvement can be expected in a few days after treatment. CXL can be considered as an adjunct therapy as soon as melting appears in severe corneal ulcers not responding to conventional, intensive treatment.

Keywords: Corneal melting, Corneal cross-linking, Corneal ulcer.

How to cite this article: Al-Sabai N, Koppen C, Tassignon MJ. UVA/Riboflavin Cross-Linking as an Alternative Treatment for Therapeutic Keratoplasty in Corneal Melting. Int J Keratoco Ectatic Corneal Dis 2012;1(1):61-65.

Source of support: Nil

Conflict of interest: None declared

\section{INTRODUCTION}

Corneal melting, due to ulcerative keratitis, has a devastating impact on ocular tissue and is a sight threatening condition. Corneal melting develops as a result of activation of proteolytic enzymes that digest human collagen, thereby facilitating stromal tissue degradation and eventually leading to corneal perforation. Several external eye diseases may result in a persistent epithelial defect that may progress to stromal melting. In a series by Killingsworth et al, 33\% of patients with persistent epithelial defects underwent a therapeutic keratoplasty. ${ }^{1}$ The underlying disease may be variable but the common factor is, in all cases, the inability to maintain a normal ocular surface and tear film. Donnenfeld et al classified their cases in three subclasses: neurotrophic keratitis, exposure keratitis and dry eye. ${ }^{2}$ The prognosis for survival of the graft is often poor under these circumstances and the goal of an emergency keratoplasty is therefore more often tectonic than optical.
This paper reports on the use of UVA/riboflavin CXL on four patients suffering from keratitis with melting and impending perforation, whereby an emergency keratoplasty was avoided in two of the four cases.

In 2003, Wollensak et al introduced the UVA/riboflavin CXL procedure to improve the biomechanical characteristics of the corneal stroma in order to stabilize progressive keratoconus. ${ }^{3}$ Based on in vitro tests on porcine cornea, Spoerl et al demonstrated that CXL augments the stromal resistance against enzymatic digestion by pepsin and collagenase. ${ }^{4}$ Spoerl hypothesized that CXL changes the tertiary structure of the collagen fibrils preventing access of the proteolytic enzymes to their specific cleavage sites by steric hindrance. ${ }^{4}$

In 2000, Schnitzler has already published his experience on applying CXL on four patients with corneal melting. He was successful in preventing perforation in three out of the four patients treated. ${ }^{5}$ It is common knowledge that UV irradiation has an antimicrobial effect by irreversibly damaging the RNA and DNA of microorganisms and their cell membranes. ${ }^{6}$ Based on this double antimicrobial action of UV light (and thus of CXL), Iseli, Makdoumi and recently Ehlers, successfully applied CXL to treat cases of infectious melting and nonhealing corneal ulcers resistant to conservative treatment, thereby preventing emergency keratoplasty. ${ }^{6-8}$

\section{CASE REPORTS}

\section{Case 1}

An otherwise healthy 44-year-old Caucasian male was referred to our department for a severe ulcerative keratitis in the right eye. According to his referral documents, the patient had a trauma on the right side of his face associated with cataract and laceration of the upper eyelid several years ago. The patient had cataract surgery complicated by epithelial ingrowth in the anterior chamber, and a surgical repair with an incomplete restoration of the upper eyelid. The left eye was without any ocular problem.

The patient presented with a history of pain and hyperemia of the right eye since 3 weeks. Best corrected visual acuity was hand movement. The eye showed pronounced ciliary and conjunctival injection, diffuse corneal edema with an inferonasally located epithelial defect with underlying infiltration and a moderate anterior chamber 


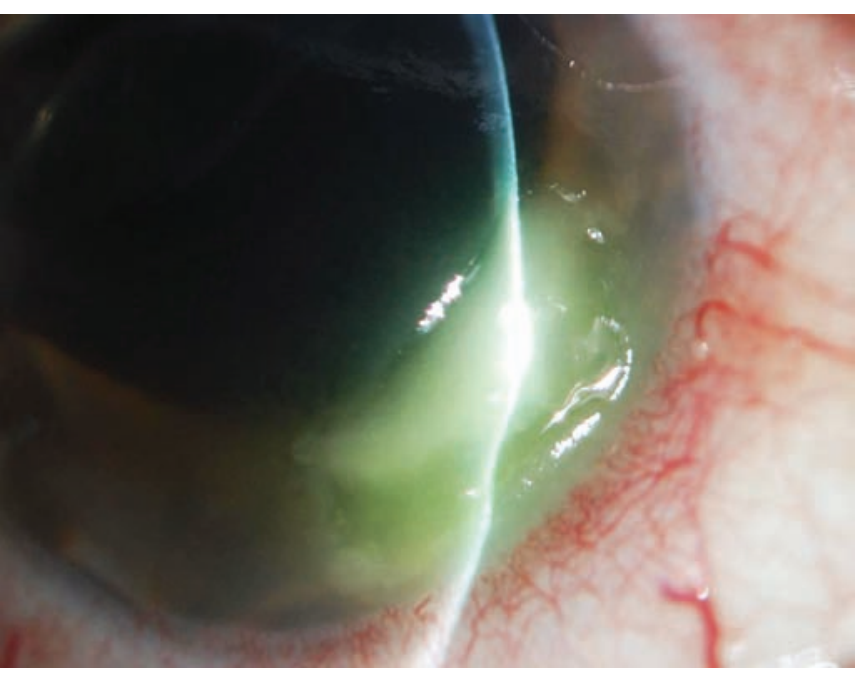

Fig. 1: Case 1: Precross-linking—an imminent perforation

reaction (Fig. 1). The location of the corneal ulcer corresponded to a notch in the upper eyelid.

The patient was hospitalized for intensive treatment with topical fortified antibiotics (tobramycin and vancomycin) every 2 hours. In the course of the following 4 days, the melting progressed to such an extent that we decided to treat the patient with CXL to avoid corneal perforation. Photosensitizing drops containing $0.1 \%$ riboflavin in dextran 20\% (Peschke Meditrade, Switzerland) were applied on the cornea, once every 3 minutes for 30 minutes. Radiant energy for the CXL treatment was UVA $365 \mathrm{~nm} 3 \mathrm{~mW} / \mathrm{cm}^{2}$ for 30 minutes using the VEGA crosslinker light source (VEGA CBM X-Linker ${ }^{\circledR}$, Ofta Hi-tech, Italy), while instillation of riboflavin was continued. After the CXL treatment, fortified antibiotics were replaced with unpreserved ofloxacin (Trafloxal EDO, Tramedico, Netherlands), dexamethasone (Dexamethasone Monofree, Thea, France) and autologous serum drops. To prevent dryness due to the defect in the eyelid and to encourage the healing process, a moist chamber bandage was also used.

Subjective improvement and signs of healing started from the 3rd day post-CXL. A corneal scraping that had been sent for microbiological examination could not identify any microorganisms. After 10 days, the eye was quiet and the corneal lesion had healed (Fig. 2).

\section{Case 2}

A 69-year-old male presented to the emergency department of our hospital with a history of pain in the right eye since more than 2 weeks. Relevant in the ophthalmic history is the fact that the patient had undergone corneal transplantation twice and a trabeculectomy as a result of complicated cataract surgery. He was still on topical steroid drops twice daily, because of persistent ocular inflammation.

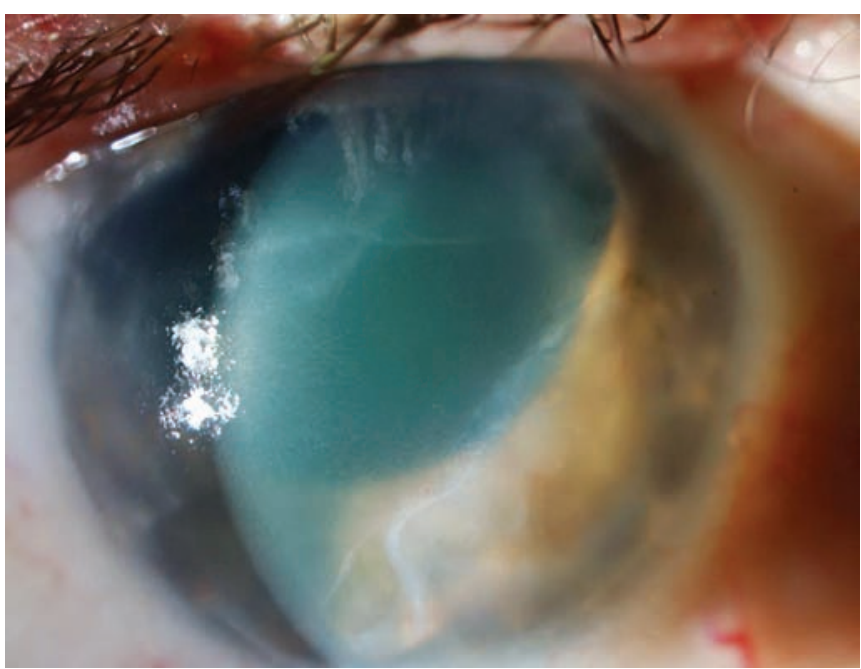

Fig. 2: Case 1: One month after cross-linking - a well-healed lesion

Best corrected visual acuity in the right eye was light perception temporally. The cornea was edematous and inferonasally the running suture had cheese-wired through the cornea causing an epithelial defect and stromal thinning (Fig. 3). A mild anterior chamber reaction was present. The ocular tension was within normal limits. Subtotal excavation of the optic nerve disk was known as a consequence of glaucoma. Examination of the left eye was completely normal.

The cornea was so thin that the risk of perforation was estimated to be real so that we decided not to remove the corneal sutures at first but to treat the patient with CXL in an attempt to stop the corneal melting. The CXL procedure was performed in the same way as specified above. After the CXL treatment, topical medication consisted of unpreserved fluoroquinolone eye drops four times a day and a moist chamber bandage. The condition stabilized and signs of improvement and healing were observed from the

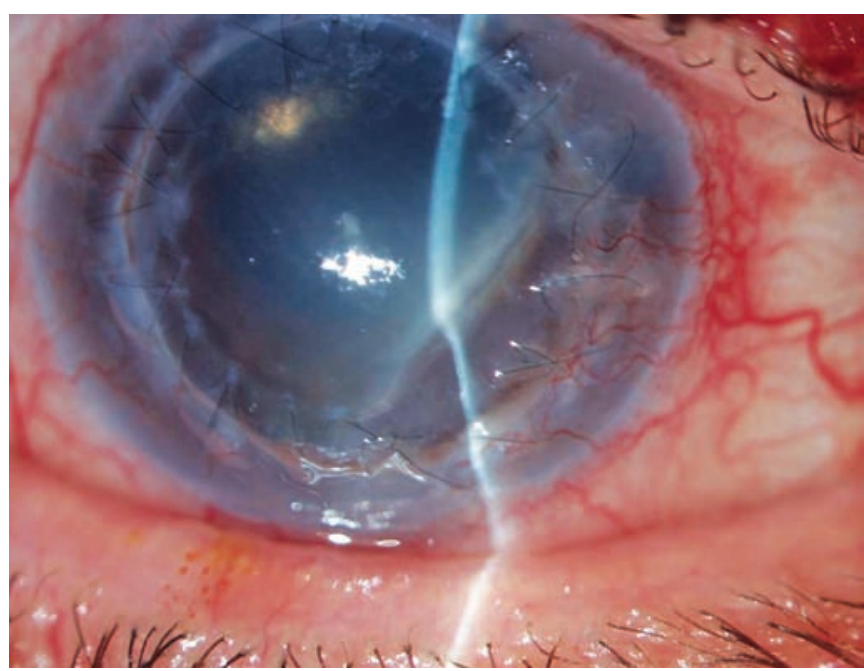

Fig. 3: Case 2: Precross-linking —melting of the cornea where the suture has cheesewired through the tissue 


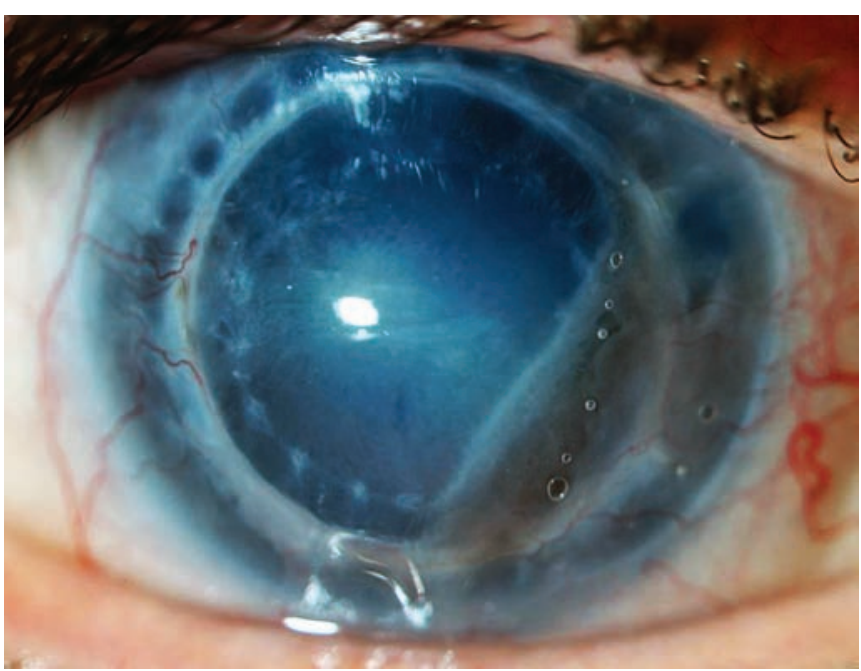

Fig. 4: Case 2: One month after cross-linking, the cornea healed and the melting was stopped

3rd day post CXL. Five days later, no epithelial defect could be seen anymore and the sutures were removed. After one month, the patient was symptom free and the cornea healed (Fig. 4).

\section{Case 3}

A 68-year-old male was seeking advice for treatment of protracted corneal ulceration in the left eye. The patient underwent penetrating keratoplasty twice in 5 months time in order to treat a corneal scar of unknown origin. The topical treatment consisted of steroid and antibiotic drops, 8 times a day.

On presentation, the best corrected visual acuity in the left eye was light perception. The cornea was edematous with a corneal infiltrate and stromal thinning (Fig. 5). Evaluation of the anterior chamber was impossible due to the corneal edema. B-scan ultrasonography of the left eye showed an attached retina with an otherwise echographically empty vitreous body. The ocular tension was within normal limits on digital evaluation.

The patient was hospitalized for intensive treatment consisting of a topical fortified antibiotic regimen of tobramycin and vancomycin every hour combined with atropine eye drops, 3 times a day and oral aciclovir $800 \mathrm{mg}$ (Zovirax, Glaxosmithkline Great Britain), 5 times a day.

In the course of the following 15 days of intensive treatment, the pain persisted and the ulceration increased in size. The dosage of fortified antibiotics had been tapered to avoid undesired toxic side-effects. Corneal scraping and PCR was performed but proved to be negative for bacterial and viral antigens. Unfortunately, no signs of healing of the ulcer could be observed and we decided to treat the patient with CXL in an attempt to stop the corneal melting. After the CXL treatment, topical medication consisted of

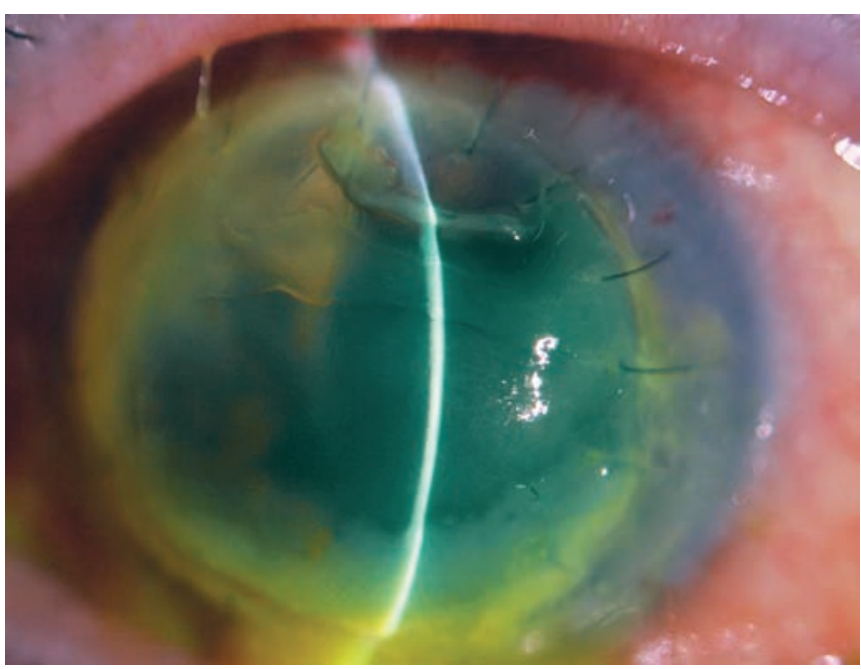

Fig. 5: Case 3: Precross-linking-corneal melting with epithelial defect

unpreserved corticosteroid and antibiotic drops, 3 times a day, intensive lubrification every hour and oral aciclovir $800 \mathrm{mg}, 5$ times a day. Despite this treatment, the corneal melting progressed and a corneal transplantation was done 1 week after the CXL.

\section{Case 4}

A 60-year-old female patient was referred for severe ulcerative keratitis in the right eye due to therapy resistant Acanthamoeba, which was cultured from a corneal scraping. For this reason, she was on topical antibiotics and corticosteroids twice a day, hexamedine (desomedine $0.1 \%$ Chauvin Laboratoire, France) and chlorhexidine 0.04\%, 8 times a day, autologous serum drops every hour, and oral ketoconazol (Nizoral 200 mg, Janssen Cilag, Belgium), twice a day.

Relevant in the ophthalmic history is the fact that the patient has worn soft contact lenses since 20 years. The left eye was without any ocular history.

At the first examination, the right eye could only distinguish hand movement. It showed pronounced ciliary and conjunctival injection. There was diffuse corneal edema with a central corneal ulceration and stromal infiltrate (Fig. 6). Evaluation of the anterior chamber was impossible due to the corneal edema. The ocular tension was within normal limits on digital evaluation. Examination of the left eye was completely normal.

Since the patient had already been hospitalized elsewhere and treated intensively with topical antiseptic eye drops, we decided to treat the patient concurrently with CXL, in an attempt to promote the healing of the keratitis and eradicate the Acanthamoeba parasites. CXL was performed in the same way as in the above-mentioned cases. After the CXL treatment, topical medication consisted of desomedine 


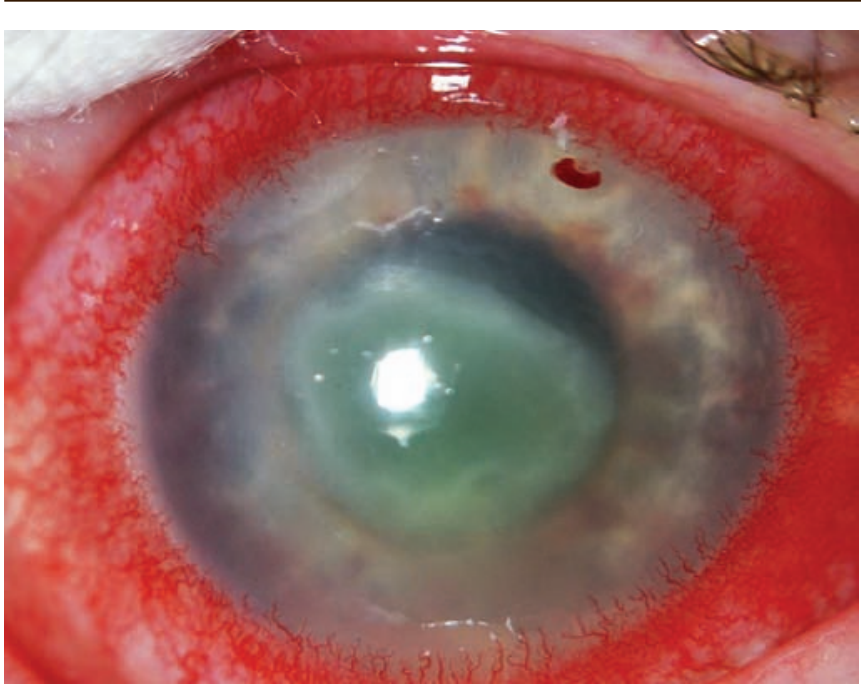

Fig. 6: Case 4: Precross-linking-Acanthamoeba keratitis

0.1\% (Hexamedine, Chauvin laboratoire, France) and chlorhexidine $0.02 \%, 6$ times a day and intensive lubrification with autologous serum drops every hour. There was no improvement in the following weeks even with increased dosage of the antiseptic medication every 30 minutes and addition of propamidine isethionate (Brolene, SonfiAventis, France) and polyhexamethylene biguanide $0.02 \%$. The condition stabilized only after performing a penetrating corneal transplantation.

\section{DISCUSSION}

Corneal melting or keratolysis is a pathological condition characterized by progressive loss of corneal tissue due to collagen degradation by proteolytic enzymes, such as metalloproteinases. ${ }^{9}$ Corneal melting is usually seen in immune and inflammatory mediated conditions, such as infections, rheumatoid arthritis, chemical burns, the use of topical eye drops like nonsteroidal anti-inflammatory drugs and steroids. ${ }^{10-12}$ It has been also reported in cases with nonabsorbable sutures and keratitis sicca. ${ }^{9,13}$

Our case series of corneal melting describes four chronically diseased eyes associated with eyelid and anterior segment pathologies. All four patients presented with a subacute ulcerative keratitis, clinically suspected for infectious keratitis, although only in the 4th case an infectious cause was established. We believe that there was not a single cause responsible for the melting. In the 1st case, exposure keratopathy in association with corneal hypoesthesia was most probably the trigger factor. In the 2nd case, the presence of the sutures and the chronic use of corticoids and the relative hypoesthesia after corneal grafting were promoting melting. In the 3rd case, possibly herpes infection contributed to the progression of the melting. In the 4th case, the persistent epithelial defect and the prolonged use of the topical toxic antiseptic eye drops played role in aggravating the melting.
Despite the use of fortified antibiotics, antiviral and antiseptic medications, the condition of the corneas worsened. The toxic effect of the intensive topical antibiotics treatment might have exacerbated the proteolytic enzyme activity. In the 2nd case, due to the imminent risk of perforation, we did not remove the sutures till the cornea was stabilized by the CXL. In case 3, the complexity of the pathology and the herpes infection might be the reason why the CXL had no effect. In case 4, due to the long period of infection before the CXL, the Acanthamoeba parasites might have found access to the deep layers of the cornea, where the CXL is less efficient. In all four cases, a breakdown of the normal corneal defense and repair mechanisms, due to a longlasting disease, were decisive in the development of melting.

With all its major drawbacks, an emergency keratoplasty 'à chaud' seemed to be the only solution as the corneal melting progressed and an imminent corneal perforation was to be feared. The risk of reinfection and rejection was enhanced by the peripheral location of the melting which would have required a large eccentric graft. The successful case reports of Iseli, Makdoumi and Ehlers, ${ }^{6-8}$ on the use of CXL for the treatment of nonresponding corneal ulcerations on intensive antibiotic treatment regimens, encouraged us to apply this treatment on our four patients.

Several studies revealed that UV irradiation can cause irreversible damage to the RNA and DNA of viruses, bacteria and parasites preventing them from further growth and replication. In addition, the oxygen radicals interfere with cell membrane integrity. We can use to our benefit this combined antimicrobial effect of UV light and free oxygen radicals. The beneficial effect of using the combined antimicrobial effect of UV light and free oxygen radicals was verified by Martins, Makdoumi and Schrier, who have tested the antimicrobial efficacy of riboflavin and ultraviolet light on bacterial and fungal isolates. The results of their tests brought them to conclude that riboflavin and UVA are very effective in the eradication of different types of common corneal pathogens. ${ }^{14-16}$

Thus far, few case reports have been published describing the successful treatment of ulcerative keratitis with the help of CXL. ${ }^{5-8,17,18}$ In most published cases, CXL was performed only after failure of medical treatment. We believe that CXL could be useful in the coming future not only for the therapy resistant cases but also as an adjunct therapy for infectious and noninfectious ulceration of the cornea and to avoid thinning and melting of the stromal tissue. CXL could provide many advantages, such as avoiding problems of antibiotic-related toxicity, bacterial resistance to antibiotic medications, hospitalization and absenteeism. Our case series confirms previous findings that CXL is a therapeutic option that has to be considered as an adjunct in the treatment of ulcerative keratitis. 


\section{REFERENCES}

1. Killingsworth DW, Stern GA, Driebe WT, Knapp A, Dragon DM. Results of therapeutic penetrating keratoplasty. Ophthalmology 1993;100:534-41.

2. Donnenfeld E, Solomon R, Perry A. Cornea. Therapeutic keratoplasty 2011;2:141:1596.

3. Wollensak G, Spoerl E, Seiler T. Riboflavin/UVA-induced collagen cross-linking for the treatment of keratoconus. Am J Ophthalmol 2003;135:620-27.

4. Spoerl E, Wollensak G, Seiler T. Increased resistance of crosslinking cornea against enzymatic digestion. Current Eye Research 2004;29:35-40.

5. Schnitzler E, Spoerl E, Seiler T. Bestrahlung der Hornhaut met UV-Licht und Riboflavingabe als neuer Behandlungsversuch bei Einschmelzenden Hornhautprozessen, erste Ergebnisse bei vier Patienten. Klin Monatsbl Augenheilkd 2000;217:190-93.

6. Iseli HP, Thiel MA, Hafezi F, Kampmeier J, Seiler T. Ultraviolet A/riboflavin corneal cross-linking for infectious keratitis associated with corneal melts. Cornea 2008;27:590-94.

7. Ehlers N, Hjortdal J, Nielsen K, Sondergaard A. RiboflavinUVA treatment in the management of edema and nonhealing ulcers of the cornea. J Refract Surg 2009;25:S803-06.

8. Makdoumi K, Mortensen J, Crafoord S. Infectious keratitis treated with corneal cross-linking. Cornea 2010;29:1353-58.

9. Allan J, Flach. Corneal melts associated with topically applied nonsteroidal anti-inflammatory drugs. Tr Am Ophth Soc 2001; 99:205-12.

10. McKibbin M, Isaacs JD, Morrel AJ. Incidence of corneal melting in association with systemic disease in the Yorkshire region, 1995-97. Br J Ophthalmol 1999;83:941-43.

11. Kuckelkorn R, Redbrake C, Reim M. Tenonplasty: A new surgical approach for the treatment of severe eye burns. Ophthalmic Surg Lasers 1997;28:105-10.

12. Guidera AC, Luchs JI, Udell IJ. Keratitis, ulceration and perforation associated with topical nonsteroidal antiinflammatory drugs. Ophthalmology 2001;108:936-44.

13. Stokes J, Wright M, Ramaesh K, Smith C, Dhillon B. Necrotizing scleritis after intraocular surgery associated with the use of polyester nonabsorbable sutures. J Cataract Refract Surg 2003;29:1827-30.

14. Martins SA, Combs JC, Noguera G, Camacho W, Wittmann P, Walther R, et al. Antimicrobial efficacy of riboflavin/UVA combination (365 nm) in vitro for bacterial and fungal isolates: A potential new treatment for infectious keratitis. Invest Ophthalmol Vis Sci 2008;49:3402-08.

15. Schrier A, Greebel G, Attia H, Trokel S, Smith EF. In vitro antimicrobial efficacy of riboflavin and ultraviolet light on Staphylococcus aureus, methicillin-resistant Staphylococcus aureus and Pseudomonas aeruginosa. J Refract Surg 2009;25: 799-802.

16. Makdoumi K, Bäckman A, Mortensen J, Crafoord S. Evaluation of antibacterial efficacy of photo-activated riboflavin using ultraviolet light (UVA). Graefes Arch Clin Exp Ophthalmol 2010;248:207-12.

17. Micelli Ferrari T, Lezappa M, Lorusso M, Epifani E, Micelli Ferrari L. Escherichia coli keratitis treated with ultraviolet A/riboflavin corneal cross-linking: A case report. Eur J Ophthalmol 2009;19:295-97.

18. Morén H, Malmsjö M, Mortensen J, Ohrström A. Riboflavin and ultraviolet-A collagen cross-linking of the cornea for the treatment of keratitis. Cornea 2010;29:102-04.

\section{ABOUT THE AUTHORS}

\section{N Al-Sabai (Corresponding Author)}

Department of Ophthalmology, Antwerp University Hospital Wilrijkstraat 10, B-2650, Edegem, Belgium, e-mail: alsabay2004@yahoo.com

\section{Koppen}

Department of Ophthalmology, Faculty of Medicine and Health Sciences, University of Antwerp, Universiteitsplein, Wilrijk, Belgium

\section{MJ Tassignon}

Department of Ophthalmology, Faculty of Medicine and Health Sciences, University of Antwerp, Universiteitsplein, Wilrijk, Belgium 